Stoa

Vol. 11, no. 21, 2020, pp. 4-29

ISSN 2007-1868

\title{
EL DEVENIR DE LA ESFERA PERSONAL Y EL PANENTEÍSMO EN LA METAFÍSICA TARDÍA DE MAX SCHELER
}

The Becoming of the Personal Sphere and Panentheism

in Max Scheler's Later Metaphysics

\author{
J. EDWARD HACKETT \\ Savannah State University \\ hackettj@savannahstate.edu
}

RESUMEN: En este artículo, explico la visión de Scheler sobre Dios y las personas desde su período fenomenológico inicial y la transición que ocurre en su pensamiento alrededor de 1922 hasta su último trabajo publicado en 1928, El puesto del hombre en el cosmos. En ese trabajo, evalúo las afirmaciones metafísicas sobre el Espíritu y el impulso compenetrantes y me insto a ver la interacción entre ambos como un ejemplo de teísmo dipolar en lugar de la tendencia a interpretar el pensamiento de Scheler como una forma de dualismo. De hecho, muestro que la explicación relacional del proceso de Scheler sobre el Espíritu y el impulso debe leerse a través de la filosofía del organismo de Alfred North Whitehead, aunque no doy a esta posibilidad interpretativa un tratamiento o defensa exhaustivo.

PALABRAS CLAVE: Whitehead - Scheler - Espíritu - Impulso · Impulso vital · Esfera personal.

ABSTRACT: In this paper, I explain Scheler's view about God and persons from his initial phenomenological period and the transition that occurs in his thought around 1922 up into his last work published in 1928, The Human Place and the Cosmos. In that work, I assess the metaphysical claims about co-penetrating Spirit and Impulsion and urge us to see the interaction of Spirit and Impulsion as an example of dipolar theism rather than the tendency to interpret Scheler's thought as a form of dualism.

Recibido el 10 de noviembre de 2019

Aceptado 30 de noviembre de 2019 
Indeed, I make the gesture that Scheler's process-relational account of Spirit and Impulsion should be read through Alfred North Whitehead's philosophy of organism though I do not give this interpretive possibility exhaustive treatment or defense.

KEYWORDS: Whitehead · Scheler · Spirit · Impulsion · Life-Drive · Personal Sphere.

\section{La intuición fenomenológica y la esfera personal}

Antes de explicar la estructura subyacente del panenteísmo de Scheler, quisiera aprovechar para explicar qué implica el método fenomenológico entendido por él y cómo este compromiso fenomenológico, aunque nunca abandonado en espíritu, abrió sus esfuerzos iniciales para caracterizar los actos religiosos y la experiencia religiosa en general. La fenomenología de la religión de Scheler es la base desde la cual su concepción del proceso de lo divino se originó en su ulterior obra, El puesto del hombre en el cosmos (1928). Por lo tanto, es un comienzo necesario.

El término de Scheler para la región de la conciencia en donde acaece la proyección intuitiva y la entrega es "La esfera" (die Sphäre). A lo largo de este ensayo, llamaré a este espacio inmanente de conciencia, la región misma de la conciencia en la que se recoge la intuición como la esfera personal; también he usado este término para referirme a la actividad de la persona en Edgar Sheffield Brightman.

Para Scheler, el proyecto fenomenológico está en contra del procedimiento husserliano de agrupar fenómenos y dejar que estos se muestren. De esta manera, Scheler pensó que la fenomenología era una actitud (Einstellung), un punto de vista desde el cual uno encuentra y aprehende una esencia dada en la inmanencia de la conciencia de principio a fin. "La experiencia fenomenológica es al mismo tiempo una experiencia "inmanente". ${ }^{2}$ Por el contrario, Husserl lo consideró como un método. Como Manfred Frings explica que para Scheler los fenómenos "están puestos entre paréntesis por la intuición, no fuera de ella" 3 En consecuencia, la fenomenología es la ampliación y purificación de la intuición inmediata en la conciencia inmanente. El fenomenólogo está, entonces, trabajando en la inmanencia de la intuición inmediata de un fenómeno en la esfera personal abstraído y demostrado "aisladamente de todo lo demás." Según Scheler, "sólo lo que es intuitivamente en un acto de

\footnotetext{
${ }^{2}$ Max Scheler, Formalism in Ethics and Non-Formal Ethics of Value. A New Attempt Toward the Foundation of an Ethical Personalism, Northwestern University Press, Evanston, 2006, p. 51.

${ }^{3}$ Manfred S. Frings, The Mind of Max Scheler, Marquette University Press, Milwaukee, 2001, p. 125.

${ }^{4}$ Ibid., p. 50.
} 
experimentar (incluso si esta esencia debe apuntar a un contenido más allá de sí mismo) ... puede pertenecer a él." ${ }^{5}$ Del mismo modo, las esencias (Wesenschau) discernidas a través de la intuición inmediata apuntan a lo que es real: las esencias que constituyen todos los actos intencionales. Pongamos un ejemplo de dónde esto llevó a los primeros esfuerzos de Scheler.

El personalismo ético de Scheler se basa en permitir la primera comprensión inicial de la aprehensión intuitiva de un fenómeno con el que entramos en relación en los actos de sentimiento de la conciencia. "El puesto actual de toda la cognición del valor o la intuición del valor (Wert-Erschauung) se destaca en el sentimiento [actos], básicamente en el amor y el odio, así como en las interconexiones de valores". 6 Por el contrario, tal aprehensión y discernimiento de la estructura de ser un acto en Heidegger siempre estaría mediada por un horizonte interpretativo. En Scheler, "toda experiencia no fenomenológica es en principio una experiencia a través de símbolos o por medio de ellos y, por lo tanto, una experiencia mediada que nunca da la cosas 'en sí mismas'." Él continúa: Solo la experiencia fenomenológica es, en principio, no simbólica y, por lo tanto, capaz de realizar todos los símbolos posibles."7 Para Scheler, la ética material a priori es anterior a la lente interpretativa a través de la cual Heidegger limitó la fenomenología. Scheler pone este contraste de fenomenología más directamente:

... fenomenología no es el nombre de una nueva ciencia ni un sustituto de la palabra filosofía; es el nombre de una actitud de visión espiritual en la que uno puede ver o experimentar algo que de otro modo permanece oculto, es decir, un reino de hechos de un tipo particular. Digo actitud, no método. Un método es un procedimiento dirigido a objetivos para pensar acerca de hechos... antes de que hayan sido arreglados por la lógica, y, en segundo lugar, de un procedimiento del ver ...Lo que se ve y se experimenta solo se da al ver y experimentar el acto mismo, en su actuación; aparece en ese acto y sólo en él. ${ }^{8}$

En cambio, revelar los actos esenciales en la aprehensión intuitiva activa y vibra la esfera personal, la base misma del devenir de la persona completa; esta vibración de la esencia, lo que Scheler llamará su funcionalización, irradia hacia afuera en la ejecución misma del acto. El cumplimiento de la esencia es, entonces, el mismo ser-en-un-acto que se dispara hacia afuera saliendo de la

\footnotetext{
${ }^{5}$ Ibid., p. 51.

${ }^{6}$ Ibid., p. 68.

${ }^{7}$ Ibid., p. 51.

${ }^{8}$ Ibid., p. 138.
} 
esfera personal. De esa manera, toda la persona se revela en lo que podríamos llamar vida-en-espíritu. Más acertadamente, la fenomenología describe la esfera personal de los actos en los que las personas viven su propio ser en la ejecución de esos actos.

Para Scheler, de acuerdo con el ejemplo de su ética, los valores son aprehendidos antes de pensar y actuar. Son pre-racionales y constituyen el campo de la esfera personal de cada persona. Así, la intuición fenomenológica destila muy claramente la primera comprensión intuitiva real y la sigue mediante la descripción de la plenitud y el alcance de estas estructuras fenomenológicas a medida que se revelan en la inmanencia de la conciencia en tipos específicos de experiencia, no solo en la experiencia de los valores. ${ }^{9}$ Por lo tanto, ahora estamos en condiciones de comprender que Scheler pudo extender este procedimiento fenomenológico para revelar diferentes estructuras inmanentes y contenidos de la experiencia en la experiencia religiosa. En el ejemplo de su ética, entonces, su ética fenomenológica desarrollará el ordo amoris como el sitio mismo de lo inmanente, mientras que los actos religiosos tendrán una estructura completamente diferente. Incluso a partir de 1913-1916, mientras escribe y publica diferentes partes de lo que se convertiría en su obra maestra, El formalismo en la ética, comenta cómo las diversas clasificaciones ordenadas de los actos y valores de sentimientos intencionales se correlacionan con esos sentimientos y abren la temática de lo Santo como una forma específica de entrega para ser investigada. En otras palabras, la razón por la que comencé a explicar la fenomenología de la ética en relación con la fenomenología de la experiencia religiosa es la transición abierta por la entrega de lo Santo:

... el acto mediante el cual originalmente aprehendemos el valor de lo sagrado es un acto de un tipo específico de amor ...en esencia, el acto se dirige hacia las personas, o hacia algo de la forma de un ser personal, sin importar qué contenido o qué "concepción" de personalidad está implícito. La autovaloración en la esfera de los valores de lo 'sagrado' es, por lo tanto, por necesidad esencial, un “valor de la persona". ${ }^{10}$

Cuando uno lee a Scheler, es posible que se desee observar una transición en la década de 1920 a preocupaciones más metafísicas y sociológicas en lu-

\footnotetext{
${ }^{9}$ Soy ambivalente sobre la gama de experiencias fenomenológicas en este punto. Sería ingenuo descartar sus clasificaciones de valor ordenadas como una experiencia más. De alguna manera, la experiencia de valor establece la posibilidad de todas las experiencias posteriores. Esta idea se centra exactamente en lo primordial uno cree que son para la persona los sentimientos de amar y odiar. ${ }^{10}$ Scheler, Formalism, p. 109. Las cursivas pertenecen a Scheler.
} 
gar de la fenomenología. De hecho, la pregunta “Qué es la persona?” Tiene todas las características del deseo de proporcionar una respuesta metafísica para no analizar la esfera personal fenomenológicamente a través de la descripción. A pesar de esta transición a sus trabajos posteriores, Scheler nunca abandona en mi lectura la lente fenomenológica de sus esfuerzos anteriores, tal como vemos en el pasaje anterior. La manera misma en que las personas viven y llenan los valores de lo Santo implica una dirección hacia la unicidad y el ser absoluto de otra persona, y este es el contenido experiencial de tal entrega independientemente de la concepción metafísica disponible sobre ese contenido experiencial. Sin embargo, también podemos comenzar a ver el espacio inicial para especular sobre esas mismas concepciones que plagan la atención de Scheler a cuestiones más metafísicas en la década de 1920 a la que se dirige Sobre el eterno del hombre, publicado en 1921. La fenomenología establece el camino hacia estas preocupaciones al describir estas diversas esencias reveladas en la conciencia inmanente, dividiendo el camino hacia las preocupaciones en sociología del conocimiento, teorías de la comunidad y antropología filosófica aún por venir.

\section{Actos religiosos en la esfera personal}

Para Scheler, los actos religiosos son un tipo de intencionalidad al igual que los actos de sentimiento intencional siempre se relacionan con una cualidad de valor. Sin embargo, a diferencia de ellos, los actos religiosos no toman objetos en el mundo típico como su correlato. Una consecuencia práctica y teórica es, entonces, que la experiencia de Dios y el devenir de esa experiencia en el acto religioso son el mismo punto de partida para investigar lo Divino. Nuestros conceptos metafísicos de Dios, entonces, comienzan en un tipo de experiencia/ experimentando. Volviendo a la singularidad de los actos religiosos, también se podría interpretar que los valores son un tipo diferente de entrega fenomenológica tanto como lo Divino lo es para los actos religiosos. Deseo dejar de lado ese tema por el resto de este trabajo. Por ahora, los actos religiosos difieren de otros tipos de experiencias. Como dice Scheler, "Nos limitaremos en general a examinar el acto religioso con simples detalles, en lugar de una descripción fenomenológica completa de la religión." ${ }^{11}$ Estas diferencias consisten en tres formas.

\footnotetext{
${ }^{11}$ Scheler, De lo Eterno en el hombre, Traducción Publishers, p. 162.
} 
(1) $\mathrm{Al}$ trascender los objetos contingentes en el mundo, los actos religiosos pueden unificar "todos los significados de las entidades del mundo en un todo."12 De esta manera, los objetos y aspectos del mundo contingente se alejan de la vista y dejan que lo Absoluto penetre el campo de la conciencia inmanente. Esta penetración es una ruptura, una ruptura en la continuidad del mundo sensual de los objetos. De hecho, nada en el horizonte del campo perceptual puede proporcionar el sentido o significado descubierto en los actos religiosos. Por lo tanto, una fenomenología de la experiencia religiosa debe comenzar aquí con esta ruptura del sentido de contingencia de la esfera personal y la escasez de la singularidad absoluta de contingencia. ${ }^{13}$

(2) Luego, al provenir de lo absoluto, una fuente extra-mundana se identifica ahora con el acto religioso, y el acto religioso sólo encuentra su posible cumplimiento en una fuente extra-mundana (posiblemente Divina) que, "en la experiencia del acto, excluye la posibilidad de entidades finitas que tengan esa función." ${ }^{14} \mathrm{El}$ mundo y el ego no son dónde se encuentran las ideas de lo Santo, incluso desde el Formalismo, Scheler escribió que "una idea de valor a priori de lo divino no tiene fundamento en la existencia de un mundo y un ego," 15 y en cambio, Scheler intenta demostrar que la idea de lo divino no presupone fenomenológicamente ninguna experiencia inductiva o histórica. Los ámbitos inductivos e históricos pueden filtrar tales ideas sobre lo Divino, pero los valores o ideas de lo Divino se nos dan primero en actos religiosos, antes de aquellos ámbitos. Por esta razón, como William James, debemos demarcar la experiencia religiosa como una forma separada de experimentar actos religiosos además de todas las otras experiencias. En comparación, en la ética de Scheler, el valor más alto de la persona se da en los valores sagrados y en el acto de sentimiento espiritual de amor. Tal calidad de valor no es reducible a ninguna otra experiencia de valor, a pesar de mi sentido a menudo ampliado del término "moral" o "ética" que incluye, por así decirlo, la creencia de Scheler en el valor absoluto de esta o aquella persona,

\footnotetext{
${ }^{12}$ Frings, The Mind of Max Scheler, p. 130.

${ }^{13}$ Esta ruptura podría ser el origen fenomenológico de la distinción de Tomás de Aquino entre contingencia y necesidad.

${ }^{14}$ Frings, The Mind of Max Scheler, p. 131.

${ }^{15}$ Scheler, Formalism, p. 293.
} 
una entrega que para él siempre tiene una nueva cualidad de valor bastante diferente a otras formas de experiencia.

(3) Finalmente, los actos religiosos se interpretan en sus implicaciones negativas. Según Frings, los actos religiosos "no tienen fundamentos ni objetivos terrenales a pesar de que pueden estar motivados empíricamente." ${ }^{16}$ Honestamente, tengo algunos problemas con esta afirmación dado mi compromiso jamesiano con el empirismo radical de que todos los conceptos tienen su origen en la coordinación de la acción y terminan en percepciones particulares. Pero permítaseme por un minuto suspender esa crítica y continuar con Scheler. Lo que implica este pronunciamiento es que los actos religiosos se relacionan con algo, una fuente inefable, en términos jamesianos, el "orden invisible" así definido porque James consideraba, muy parecido a Scheler, la apertura a la que podría estar el contenido de lo Santo. Para Scheler, sin embargo, esta participación en la inefabilidad de la fuente extra-mundana corta todo el camino hacia la conciencia inmanente. Scheler operacionalizará la manera en que lo Santo se convierte en el fundamento del ser y esta explicación puede encontrarse en su último trabajo, El puesto del hombre en el cosmos.

\section{Cerrando la brecha entre la fenomenología y la antropología filosófica}

Cerrar la brecha entre la fenomenología y la antropología filosófica no es una transición fácil, es decir, la adopción del teísmo católico durante su período fenomenológico, aproximadamente desde el año 1913, hasta principios de la década de 1920. Peter Spader detalla cuántos biógrafos lamentablemente pintan la vida psicológica de Scheler como inestable, y esta inestabilidad psicológica conmina a proyectar el pensamiento de Scheler a la luz de su vida. ${ }^{17}$ Spader, como yo, considera que esta tendencia simplifica demasiado las tensiones inherentes al pensamiento de Scheler. Los detalles son complicados, por lo que sólo repetiré brevemente lo que Spader explica con mayor detalle. Primero, en Sobre lo eterno en el hombre, detalla su posición de apoyo

\footnotetext{
${ }^{16}$ Frings, The Mind of Max Scheler, p. 131.

${ }^{17}$ Peter Spader, Scheler's Ethical Personalism: Its Logic, Development, and Promise, Fordham University Press, Nueva York, 2002, pp. 176-181. Se hace especial mención a Fr. John Nota's Max Scheler: The Man and His Work and John Staude's Max Scheler: An Intellectual Portrait.
} 
al teísmo hasta la Navidad de 1922, y la nueva posición contra el teísmo se da pistas en sus Problemas de sociología del conocimiento de 1924. Scheler, argumenta Spader, nunca cambió su ética, sino que cambió sus creencias metafísicas y religiosas. ${ }^{18} \mathrm{La}$ fenomenología moral subyacente de la ética personalista será la misma, independientemente de qué andamiaje especulativo defienda lo que experimentamos. Scheler habló sobre esta ruptura y cambio de creencia del teísmo clásico a su nueva antropología en el tercer prefacio del Formalismo en la Ética en 1926. "Las ideas en este trabajo no se ven afectadas por el cambio en mi posición metafísica fundamental". Scheler continúa: "Porque nunca fue mi intención establecer en este trabajo una fundamentación de la ética sobre la base de algún tipo de presuposición acerca de la naturaleza y la existencia y la idea y la voluntad de Dios." 19 A pesar del pronunciamiento de Scheler contra el hecho de que la metafísica no es importante para la ética, la pregunta sin respuesta que Spader nunca aborda es ¿qué pasa con la metafísica personalista subyacente si Scheler pasa de una concepción personalista de Dios a una panenteísta? ¿Puede el nuevo Dios panenteísta seguir siendo fundamental para la experiencia de valor, incluso si Scheler nunca tuvo esa intención al final de su vida? Ciertamente, hay razones por las cuales Scheler se enfocó en el teísmo clásico en su período más católico de su vida. Para responder eso, debemos ir al cambio de posición de Scheler en $E l$ puesto del hombre en el cosmos que data de la época del tercer prefacio en el Formalismo en Ética.

Con respecto a Scheler, tomemos algunas breves señales de Eugene Kelly en su Introducción al Puesto del hombre en el cosmos. Hay dos puntos que vale la pena considerar. Primero, la ideación es el acto de la mente para tener ante sí la naturaleza de todas las cosas que encontramos en nuestra experiencia de ellas, y El puesto del hombre en el cosmos hace uso exclusivo del reclamo de la ideación como veremos. Segundo, Kelly insinúa que ésta es la función de la fenomenología en términos de lo que se convierte en este último trabajo enfocado en la ideación, sin embargo, no tiene importancia metafísica. Sin embargo, difiero con Kelly en este punto, ya que su definición de fenomenología no tiene que ver con la metafísica, a pesar de que existen implicaciones metafísicas al ocupar la actitud fenomenológica de Scheler. Para mí, la feno-

\footnotetext{
${ }^{18}$ Spader, Scheler's Ethical Personalism, p. 183.
}

${ }^{19}$ Scheler, Formalism, p. xxxvi. 
menología siempre entra en la ontología. ${ }^{20}$ Según Kelly, "la fenomenología es más bien la comprensión mental, la cognición de los elementos de significado que encontramos en el mundo; requiere una reflexión intuitiva sobre el contenido del significado de los términos en un esfuerzo por exhibir sus relaciones esenciales entre sí y su orden de fundamento." ${ }^{21}$ Estas cogniciones son primordiales, constitutivas y bien pueden ser lo que otros fenomenólogos han postulado como intencionalidad. Sin embargo, tan pronto como los contenidos de significado que llevan las cosas en el mundo se postulan como los términos que se encuentran en su antropología filosófica: vida, espíritu, persona, entonces hay algunos que dan por sentados los compromisos que no se cuestionan en la cognición primordial, a pesar del papel de la intuición en ella. En otras palabras, la neutralidad fenomenológica de describir la intuición inmediata de los actos inmanentes de conciencia ya está cargada de compromisos ontológicos, por lo que afirmar la falta de importancia metafísica de la fenomenología es ridículo ya que es erróneo. ${ }^{22}$

Si eres nuevo en la antropología filosófica, entonces, visitemos, por así decirlo, la entrada de Scheler en la cuestión. El término encuentra su primer uso real en Kant, aunque su versión me parece tediosa. Permítanme definirlo de una manera que pueda encontrar sinergia en Scheler y ayudarnos con nuestra experiencia compartida de lo Divino en la esfera personal. La antropología filosófica es un intento de interpretar la estructura ontológica de la persona humana en términos de la esfera personal y la relación de la esfera personal con todas las demás formas de seres. De esta manera, la esfera personal incluye dentro de sí lo que del universo y cualquier ciencia, natural o social tengamos de él y de nosotros mismos. Con referencia a las ciencias sociales, estas suponen que las personas humanas están sujetas a leyes causales que

\footnotetext{
${ }^{20}$ Una frase pronunciada por Ken Stikkers en nuestras muchas conversaciones durante la fase de escritura de la disertación. Esta perspectiva permanece en todo mi trabajo. La fenomenología es un camino hacia una metafísica que preserva la relación y el proceso de la experiencia continua. Más novedoso para mi crecimiento en los últimos años es la afirmación interpretativa adicional de que la fenomenología es una forma estrecha de filosofía de proceso. Alfred North Whitehead sostiene que todas las ocasiones y entidades reales tienen experiencia y, aunque los seres humanos tienen conciencia como una forma de experiencia, no todas las experiencias son aquellas en las que las entidades involucradas tienen conciencia

${ }^{21}$ Eugene Kelly, "Introduction” en Max Scheler's Human Place in the Cosmos, Northwestern University, Evanston, 2009.

${ }^{22}$ Esta implicación de que la fenomenología casi siempre se transforma y entra en una ontología fue el tema del libro del año pasado que publiqué sobre la fenomenología de los valores de Scheler. Véase mi Persons and Values in Pragmatic Phenomenology: Explorations in Moral Metaphysics, Vernon Press, Malaga, 2018.
} 
las ciencias pueden descubrir de la misma manera que las ciencias naturales estudian los objetos físicos. Por el contrario, la antropología filosófica de Scheler no hace que la persona humana sea simplemente otro objeto sujeto a leyes causales en cualquier interpretación científica. En cambio, esos mismos puntos de vista científicos dan por sentado los supuestos metafísicos y epistemológicos necesarios para interpretar a la persona humana como un objeto causal entre muchos otros objetos causales en las ciencias. Cuando se realiza este enfoque de solo ciencia, perdemos de vista la esfera personal como la estructura de origen que da lugar a todas las interpretaciones de la persona humana. En fenomenología, esta perspectiva a menudo se llama actitud natural.

La actitud natural no es, por así decir, problemática en sí misma. De hecho, toda investigación científica debe proyectar y probar hipótesis del mundo y sus objetos como si estuvieran en una relación causal con otros objetos. Más claramente, acepto que, en el fondo, toda investigación científica debe asumir el naturalismo metodológico, incluso si el científico en cuestión no es un naturalista ontológico. El naturalismo metodológico ocupa un punto de vista en relación con la naturaleza y asume que todas las relaciones son causales y pueden ser experimentadas. El naturalismo ontológico, por el contrario, está comprometido con la opinión de que las únicas cosas que existen son entidades en el espacio-tiempo. Sin embargo, imaginar y proyectar esta red causal de relaciones, sobre todo, ofusca sin sentido crítico la forma en que el ser humano existe en relación con el mundo. Por esta razón, los fenomenólogos siempre han intentado recuperar una concepción original de la experiencia humana (un brightmaniano podría usar frases como la experiencia de experimentar la experiencia). ${ }^{23} \mathrm{Al}$ purgar la experiencia del punto de vista en tercera persona totalmente inclusivo (y por lo tanto impersonal) de la actitud natural, Scheler describe la relacionalidad de la esfera personal en su sentido activo en primera persona. Heidegger tenía su término Dasein; Husserl tenía su ego trascendental; y Scheler tiene el término persona. Además, incluso si fuéramos idealistas personalistas como otros personalistas como Brightman,

\footnotetext{
${ }^{23}$ Pido disculpas por una frase tan extraña en inglés. El fenomenólogo está tratando de capturar el origen funcional del significado en la experiencia al mostrar a los lectores la continuidad de la experiencia, sosteniendo y prolongando una visión descriptiva del punto de vista en primera persona. Tal vez esa sea la mejor manera de decir lo mismo: la continuidad de la experiencia en curso. Sin embargo, se señala que implicará un gerundio operativo y convertirá los verbos en sustantivos para expresar la concepción fenomenológica de la experiencia que se manifiesta en el tiempo dependiendo del tipo de experiencia que se describe.
} 
un idealismo personalista basado fenomenológicamente está comprometido a preservar el mismo sentido y significado de la persona como el origen del significado que encontramos en el pensamiento de Scheler. Nos damos cuenta de significado y valor en el lenguaje y la acción como personas. Scheler hace este punto exacto al articular el alcance de las dos formas fundamentales en que las personas devienen en su antropología filosófica, "los seres vivos no son solo objetos para los observadores externos, sino que también están dotados del modo de ser-para-sí-mismos, así como de una interioridad a través de la cual también son conscientes de sí mismos."24

\section{Las tres concepciones de la persona en Scheler}

Scheler comienza su Puesto del hombre en el cosmos con tres concepciones de la persona humana. En cada concepción, entonces, puede haber un elemento de verdad. Sin embargo, estas tres tendencias interpretativas sólo se refieren al problema de la persona en Europa y la civilización europea. De esta manera, debemos permanecer abiertos a que existan otras versiones interpretativas de la persona en otros sistemas filosóficos del mundo (por ejemplo, como las interpretaciones budistas de la vida como "solo mente" o como el estado natural de ser una criatura encarnada espontánea y amorosa). Las categorías agrupadas de Scheler no son tan establecidas y rígidas como las categorías de Scheler pretenden. Para él, son irreconciliables y es exacto que están en constante tensión entre ellos a nivel cultural. Estas categorías de la persona son:

(1) La interpretación de la persona creada es el resultado de la tradición judeocristiana. Scheler no menciona que el islam contribuya a esta concepción de la persona a pesar de que es una religión abrahámica como las otras dos que acabamos de mencionar.

(2) La interpretación de la persona racional es el resultado de la pincelada de Scheler con un gran pincel de nuestra tradición griega antigua. Permítaseme reproducir todo el pasaje:

...el ser humano es lo que es a través de su posesión de lo que se llama variablemente 'razón', logos, phronesis, ratio, mens — "logos" que significa aquí la posesión del habla, así como la capacidad de captar el "qué" [la esencia] de todas y cada una de las entidades. Estrechamente conectada con esta visión está la teoría

${ }^{24}$ Max Scheler, The Human Place in the Cosmos, Northwestern University Press, Evanston, 2009, p. 7. 
de que también hay una razón por encima del ser humano que subyace en todo el universo y con la cual el ser humano solo está en un estado de participación. ${ }^{25}$

En este pasaje, participamos en un universo racional. Esta participación ontológica armoniza con el sentido más amplio u orden y propósito en ella. Por lo tanto, podemos ver cómo y por qué Scheler puede encontrar significativa la participación de personas con un propósito dado que su fenomenología discierne las relaciones ontológicas a través de las cuales aprehendemos nuestra participación desde el principio en una experiencia hasta el final de la experiencia.

(3) La interpretación naturalista de la persona encarna una concepción en la que la persona humana "representa una etapa tardía en la evolución de nuestro planeta." ${ }^{26}$ En esta concepción, las personas son producto de energías y habilidades animales que hemos heredado de nuestro pasado ancestral y evolutivo compartido. Esta visión naturalista de la persona llega acríticamente a su interpretación ontológica de la persona desde categorías científicas hasta e incluyendo teorías evolutivas que definen al ser humano como un fabricante de herramientas (homo faber) y nada más.

\section{Geist und Drang}

Antes de hablar sobre la concepción de Scheler de la persona, debería discutir brevemente por qué estamos hablando acerca de su visión sobre la persona en su antropología filosófica si estamos interesados en su concepción panenteísta de Dios. Como Scheler nunca abandonó su metodología fenomenológica que subrayaba su metafísica especulativa (más específicamente el panenteísmo como una forma de teísmo especulativo), entonces debemos observar cómo se experimenta Dios en la persona. En otras palabras, al prestar atención a cómo la persona deviene con respecto al Geist y el Drang, podremos entender la propuesta panenteísta de Scheler sobre cómo llega a ser el fundamento del Ser en su Puesto del hombre en el cosmos.

La solución de Scheler sobre la persona es más específica. En muchas de estas interpretaciones de la persona, un aspecto de ésta se juega más que otra como una naturaleza estática y parte de ella. La interpretación grecorromana de la persona racional representa la teleología y la racionalidad a expensas

${ }^{25}$ Scheler, The Human Place in the Cosmos, p. 5.
${ }^{26}$ Ibid. 
de la animalidad enfatizada en la interpretación naturalista. La solución de Scheler consiste en sintetizar entre estas categorías, interpretando las realidades biológicas y psíquicas de la vida humana en términos de convertirse en Drang, término que la erudición de Frings y Scheler definen principalmente como impulso, un proceso de impulsos que motiva, anima y subyace al movimiento y los instintos de la vida. Luego, Scheler interpreta el devenir de sublimar los impulsos con lo que él llama it Geist. Representado como espíritu, Scheler afirma: "Casi ningún otro término ha sido abusado en el pasado tanto como este, con el resultado de que solo unas pocas personas pueden pensar en algo específico cuando se utiliza el término."27 Scheler interpreta la esfera personal cuando somos dados a nosotros mismos. ${ }^{28}$ El espíritu se refiere al centro mismo de los actos en la esfera personal. En este modo de autodonación hacia nosotros mismos, se incluye la razón, pero también el pensamiento de ideas y la intuición primordial. Al hablar de esta intuición, esta es la forma más elevada de existencia personal que conecta los inicios fenomenológicos del trabajo de Scheler extendido ahora a su antropología filosófica. En su concepción se incluye la clase específica de actos volitivos y emotivos como la libre elección, dicha, desesperación (sus dos ejemplos de sentimiento psíquico en el formalismo), maravilla, asombro, arrepentimiento, amor y amabilidad. ${ }^{29}$ En este nivel, Scheler piensa que somos personas, no sólo centros psíquicos de impulsos de funcionalización concentrados, ni somos sólo animales. El espíritu se define como "el desapego existencial del ser orgánico". ${ }^{30}$ Para comprender la relación entre el espíritu y los impulsos, debo pasar a explicar los impulsos en Scheler.

Lo que hace la contribución de Scheler a la antropología filosófica es no sucumbir al cartesianismo de muchas interpretaciones religiosas. A diferencia de Descartes o la teología cristiana que niega la animalidad y los procesos de la vida, Scheler abrió su antropología filosófica a una ontología de la vida.

${ }^{27}$ Scheler, The Human Place in the Cosmos, p. 27.

${ }^{28}$ Admito que este es mi juego con el intento de Scheler de construir un sistema. Uno puede interpretar dos formas de dualismo como una forma de interaccionismo o epifenomenalismo en lugar de dos aspectos de la autodonación. Claramente, sin embargo, empujar a Scheler al proceso de pensamiento e insistir en que Drang y Geist son dos aspectos en los que nos damos a nosotros mismos, simplemente articula el proceso de nuestra visión de nosotros mismos en relación con el todo más grande del cosmos: nuestra creación de nosotros mismos en un momento y el retroceso de ese momento en otro. Ya que Scheler abandona un catolicismo directo por el panenteísmo, una visión orientada a este proceso que cumple con el llamado de Whitehead de nunca dividir la realidad en relatos bifurcados de la realidad.

${ }^{29}$ Scheler, The Human Place in the Cosmos, p. 26.

${ }^{30}$ Ibid., p. 27. 
Sin embargo, como declaró Spader, estas fuerzas están en oposición. "Geist es lo que permite que la persona humana idee; se opone al Drang, esa fuerza que es la esencia de la vida ". ${ }^{31}$ De esta manera, Scheler vio ese impulso como la materialidad primaria en la cual ocurre la espiritualización del impulso. De esta manera, Scheler nos ofrece el hecho de que las personas devienen como vida-en-espíritu, no tan opuesto como piensa Spader. Por esta razón, Scheler nos ofrece una filosofía de proceso del devenir de Dios y de las personas en lugar de una metafísica estática o lo que Heidegger pudo haber llamado una metafísica de la presencia. Manfred Frings describe la filosofía de Scheler en general, incluyendo su inicio en la década de 1920 para desarrollar una metafísica y antropología filosófica. Frings, escribe, "la filosofía de Scheler es uno de convertirse, al no ser". ${ }^{32}$ Devenir es ser y conlleva relacionarnos de nuestra parte con el todo. En otras palabras, la vida está en proceso en varias capas en las cuales el impulso está operativo y constantemente en proceso. Él divide esta manifestación operativa de la vida en varios estratos. Si bien no explico la distinción aquí, también es primordial mencionar que la distinción entre espíritu e impulso rastrea y representa lo que se convirtió en los factores ideales y reales de Scheler en su filosofía de la cultura esbozada en su Sociología del conocimiento. Por ahora, echemos un vistazo a los niveles de impulso descritos en El puesto del hombre en el cosmos.

El primer nivel de impulso psíquico ocurre en las plantas. Las plantas, especuló, solo tienen impulso hacia el crecimiento y la reproducción. La fotografía de lapso de tiempo indicó que las plantas despliegan sus hojas para maximizar la fotosíntesis. Por lo tanto, los cambios en su entorno casi requieren que las plantas cambien todo su estado de ser en relación con el medio ambiente. Las plantas no correlacionan su impulso hacia objetos específicos en el medio ambiente, sino que se relacionan con él, como un todo mundo circundante. Esta falta de especificación del objeto relacionado es la principal diferencia ontológica entre los animales y las plantas. En un nivel diferente, los animales experimentan un impulso que informa a los muchos estados orgánicos que consiguen, incluida la memoria. Tanto las plantas como los animales encuentran sus impulsos en la resistencia al mundo, y esta resistencia es la forma en que el mundo se da a todas las formas de convertirse en vida. Los animales experimentan un centro de impulso más activo en el hecho de que la sensación y la conciencia experimentan resistencia en el mundo. También es en este pri-

${ }^{31}$ Spader, Scheler's Ethical Personalism, p. 185.

${ }^{32}$ Manfred Frings, The Mind of Max Scheler, p. 263. 
mer nivel de interioridad de la vida en el que Scheler avanza la afirmación de que la unidad más concentrada de la naturaleza y su devenir se encuentra en la persona humana. ${ }^{33}$

La segunda forma de impulso en la vida se organiza en el instinto. Al rechazar las ciencias psicológicas, Scheler define el instinto solo en relación con el comportamiento de los seres vivos. Curiosamente, Scheler afirma que "el comportamiento es independiente de los movimientos cinéticos fisiológicos que lo llevan a cabo y, como tal, sus características son determinables sin referencia a los conceptos físicos y químicos de los estímulos" ${ }^{34}$. De esta manera, Scheler está tratando de evitar reduccionismos en estas partes y procesos físicos primarios a partir de los cuales la actitud natural caracterizaría estas experiencias. Al mismo tiempo, sin embargo, los hechos naturales del cuerpo pueden restringir la forma de entender el comportamiento y sus esfuerzos pueden estar abriendo un espacio en el que se está introduciendo un tipo de dualismo para los seres humanos dada nuestra ubicación única en la ontología de la vida. Sin embargo, una lectura más generosa llama la atención sobre el hecho de que Scheler quería avanzar en una interpretación del instinto que respetara el hecho de que "el comportamiento instintivo debe tener la sensación de ser útil para todo el organismo". ${ }^{35}$ Por lo tanto, la ontología de la vida respeta todo el organismo. Además, el instinto se manifiesta en relación con la constancia de los ritmos habituales de la vida. Los instintos parecen adaptativos, más abiertos y menos rígidos que cualquier metáfora de mecanismo que se tome prestada de las imágenes de las máquinas para caracterizar los procesos de la vida (por ejemplo, la mente como una computadora, el cuerpo como una "máquina bien engrasada", etc.). Por esta razón, los instintos siempre sirven a la especie. No sirven al individuo. Los instintos son, entonces, solo una forma de reaccionar a estructuras específicas fijas, no los factores cambiantes que ocurren en el medio ambiente entre los individuos. Aun así, hay un tipo de conocimiento de sentimientos que se les da a los organismos en esta etapa. Los valores son el futuro sentimiento de resistencia y las etapas iniciales de valor comienzan aquí cuando el organismo puede tener "conocimiento instintivo de atraer y repeler momentos de resistencia". ${ }^{36}$

\footnotetext{
${ }^{33}$ Scheler, The Human Place in the Cosmos, p. 10.

${ }^{34}$ Ibid., p. 11.

${ }^{35} \mathrm{Ibid}$., p. 12, (la cursiva es mía).

${ }^{36}$ Ibid., p. 16. Uno podría ver el origen de los sentimientos sensibles y la valoración de lo agradable y desagradable en este nivel de conocimiento instintivo.
} 
Hasta ahora, los impulsos y los instintos aún no se diferencian en formas de vida inferiores a las nuestras. En esta visión jerárquica, entonces, los instintos no pueden reducirse a hábitos o arcos reflejos individuales específicos. En cambio, los impulsos son formas apagadas y primitivas de la vida psíquica. Para que los impulsos emerjan del instinto, un organismo necesita formar asociaciones más complejas para tener el tipo de complejo psíquico necesario para que los impulsos se manifiesten. El dinamismo se manifiesta, entonces, en la vida animal cuando las sensaciones y las representaciones se conectan y un impulso de gratificación las busca en lugar de simplemente ser abastecidas por los ritmos de la vida.

La tercera forma de impulso ocurre en el comportamiento habitual. Para Scheler, este nivel está más allá de las plantas y solo ocurre en niveles cuyas formas anteriores fueron dirigidas por procesos de utilidad. Cuando los movimientos producidos por el hábito resultan exitosos, los impulsos se satisfacen y los animales tienden a fijarse en patrones exitosos en lugar de acciones fallidas, y en referencia al formalismo, los valores de lo agradable y desagradable se producen aquí a partir de sentimientos sensibles. Esta capacidad de formar hábitos surge de la memoria asociativa, y en los animales, las leyes de asociación son simplemente reflejos condicionados de un arco reflejo que ocurre. La afirmación más interesante de Scheler en todo esto es la compleja distribución de la memoria asociativa en todas las formas de vida animal. En los seres humanos, la memoria asociativa ocurre copiando a otros. A la dimensión biológica de la repetición de impulsos, las satisfacciones producidas por la imitación es tradición. El hecho de que seamos una especie histórica y que podamos ir más allá del recuerdo recuerda la forma más elevada de vida animal. Este movimiento más allá indica para Scheler la disolución de la tradición. Experimentarnos recordando actos hacia un evento pasado ya es la disolución de la tradición vivida. ${ }^{37}$

En la cuarta concepción de la vida psíquica, Scheler la describe como "inteligencia práctica ligada orgánicamente". ${ }^{38}$ En esta frase, vemos el compromiso de Scheler con una ontología de la vida que une nuestra inteligencia práctica y su compenetración y dependencia mutua. Nuestra inteligencia práctica está al servicio de los impulsos. Para Scheler, la inteligencia tiene dos lados. Primero, está su definición práctica, el cómo la inteligencia práctica simplemente está actuando de manera significativa sin consultar ensayos

${ }^{37}$ Ibid., p. 20.

${ }^{38}$ Ibid., p. 21, (las cursivas pertenecen a Scheler). 
pasados como lo hace un organismo en la memoria asociativa. Los seres humanos y los ratones en una exhibición de laboratorio actúan significativamente en ensayos pasados. En segundo lugar, el lado psíquico de la inteligencia es "una visión repentina del contexto de los hechos y valores dentro del entorno". ${ }^{39}$ En este nivel del Formalismo, podemos estar en un sentimiento vital anticipando valores de salud y bienestar de la persona en el medio ambiente. Aunque implícito, deduzco que tener acceso a los valores comprendidos, pero también el hecho de que representamos el entorno y la percepción "de manera anticipatoria" es una reminiscencia de los sentimientos vitales "anticipando el valor de los posibles estímulos y su llegada [valores vitales como él los llama]." 40

Revisemos en esta etapa lo que hemos hecho hasta ahora. Primero, expliqué la relación entre los primeros esfuerzos de la fenomenología de la religión, la metodología fenomenológica, y comparé esos métodos con lo que cambió históricamente en el movimiento de Scheler del teísmo clásico al panenteísmo en su texto central, El puesto del hombre en el cosmos. Luego, centramos nuestros esfuerzos en introducir la cuestión de las personas como la motivación para abrir la especulación sobre la antropología filosófica de Scheler y la metafísica posterior en la que la esfera personal se convierte en el lugar donde Dios deviene en en nosotros y a través de nosotros. En esta sección, entonces, la toma de impulso vital se convierte en la característica central de toda la unión psicofísica de la persona, y entonces fue necesario explicar el devenir del impulso vital en todos los niveles jerárquicos de la vida que Scheler describió antes de que podamos explicar cómo estos niveles de impulso vital se revelan activamente en el devenir de Dios y las personas. Explicar el proceso del devenir de Dios y las personas es ahora la tarea central de la siguiente sección.

\section{El devenir de Dios en espíritu}

Para Scheler, Dios se convierte en actos de espiritualización, y este devenir es causalmente ineficaz. Como Scheler afirmó, "inicialmente, el espíritu no tiene energía propia." ${ }^{41}$ El espíritu no puede hacer nada, pero tiene una idea del hecho de que no hay una dirección teleológica general de los actos espirituales que las personas eligen realizar en el mundo. En cambio, las personas

\footnotetext{
${ }^{39}$ Ibid., p. 2.

${ }^{40}$ Scheler, Formalism, p. 340.

${ }^{41}$ Scheler, The Human Place in the Cosmos, p. 48, (las cursivas pertenecen a Scheler).
} 
están abiertas al mundo a la revelación de valor en espíritu, pero requieren el impulso vital para que se desarrolle. Como Eugene Kelly señala, Scheler postula: "Drang como el fundamento del ser, el espíritu es Gleichursprünglich o igual en primordialidad al Drang, pero completamente sin poder para causar eventos". ${ }^{42}$ Las energías impulsoras ya deben estar presentes para establecer la condición de realización del espíritu en el mundo. Dado que lo que Scheler llama Dios aquí se convierte en Geist, Scheler está rechazando al Dios abrahámico que creó la vida ex nihilo. Por esta razón, Dios no puede hacer cambios sin que la persona humana participe para realizar a Dios. Esta participación y cooperación ontológica reside en el devenir de la fuerza vital a través de la cual el cosmos está creciendo y luchando. Dios solo puede hacer que las personas vean la bondad a través del amor. Por esta razón, estoy definiendo el panenteísmo como el espíritu de las energías de la vida a través de la libertad de aquellos seres para los cuales Dios se une a la realización de los valores. Dios se convierte en todos los seres capaces de ser personas.

En nuestro breve estudio, vimos que Scheler valora mucho al ser humano como un ser de espíritu, pero también que lo que realiza el espíritu en este mundo son los impulsos y estructuras vitales. El espíritu es un potencial dentro del movimiento de la vida, y los comentaristas de Zachary Davis a Peter Spader interpretan este doble movimiento posible como un tipo de dualismo; sin embargo, este dualismo es atípico de las variedades racionalistas que obtenemos en Descartes y Leibniz. Incluso Scheler se describió a sí mismo como un nuevo tipo de dualista. ${ }^{43}$ A pesar de este encanto del dualismo, hay algunos factores que debemos tener en cuenta al aceptar la explicación procesal de Scheler sobre las personas.

Primero, las personas siempre devienen y nunca se completan. Hay un dinamismo en la visión de Scheler en el centro mismo de ser una persona, y lo que he llamado en este trabajo la esfera personal. Los impulsos de la vida se espiritualizan y los actos espirituales se vitalizan y encarnan. De esta manera, las personas pueden tender a los valores asociados con la vida animal, o pueden llegar a auto transcenderse en la apertura y libertad inherentes a la actualización de su potencial espiritual. La esfera personal contiene dentro de sí "una estructura monárquica de actos de los cuales un acto a la vez tiene

${ }^{42}$ Eugene Kelly, Material Ethics of Value: Max Scheler and Nicolai Hartmann, Springer, Dordrecht, 2011, p. 190.

${ }^{43}$ Sobre la interpretación de Zachary Davis, véase su artículo escrito en colaboración con with Anthony Steinbock "Max Scheler," The Stanford Encyclopedia of Philosophy, Edward N. Zalta (ed.), Spring, 2019, vid. https://plato.stanford.edu/archives/spr2019/entries/scheler/. 
su función de gobierno y dirección y está dirigido a ese valor y esa idea con la que la persona, en cualquier caso, se "identifica". ${ }^{44}$ Lo que valoramos, entonces tiene una relación directa con el movimiento ontológico de la esfera personal. Aun así, hay una ley ontológica enunciada en la mitad posterior de la noción de espíritu de Scheler que se apoya en este movimiento ontológico. Con respecto a la impotencia del espíritu (lo que ya he llamado su ineficacia causal). Sobre esto, Scheler escribe: "Desde el principio, lo que es humilde es poderoso, y lo que es más elevado es impotente" ${ }^{45}$ Según Scheler, las formas más bajas de vida animal, son más dependientes de sus impulsos y los niveles de impulsos psíquicos que revisamos en la sección anterior.

Para Scheler, entonces, esta identificación es también el propósito funcional y práctico de la comprensión metafísica del ser humano. La esfera personal toma sus aspiraciones de cómo y por qué la persona se comprende a sí misma. En la mayor formación de valor, las personas son radicalmente más libres que si identifican su base de naturaleza animal común a muchas cosmovisiones naturalistas que no tienen espacio para la auto trascendencia. Scheler llamó a esto estar abierto al mundo (Welt-Offenheit) Las personas son, por lo tanto, una reserva inagotable de potencial para realizar valores cada vez más altos y más nuevos aprehendidos a través de actos de ideas espirituales. Para Scheler, este potencial actúa sobre un tipo de amor agápico que hace que la persona vea una concepción más valorada de su propia persona y una comunidad potencialmente más elevada. Más concretamente, estas concepciones de valor no apuntan a una forma específica de ser en términos de cómo participar en valores más altos, sino solo que las personas deben realizar más amor en el mundo.

$\mathrm{Al}$ aprehender estas esencias de valor para aspirar a niveles más altos de ser, la dirección ontológica de la persona corre "de abajo hacia arriba en el mundo en que vivimos". ${ }^{46}$ De esta manera, Scheler se parece mucho al Personalista de Boston Edgar S. Brightman al pensar que las religiones son sobre la experiencia del valor, tal vez mejor descrita como la realización del valor. Brightman articula esta idea con respecto al hecho de que la religión fomenta y ayuda a preservar los más altos valores de verdad, belleza y bondad. ${ }^{47}$ Tan-

${ }^{44}$ Scheler, The Human Place in the Cosmos, p. 46.

${ }^{45}$ Ibid., p. 47. (las cursivas pertenecen a Scheler).

${ }^{46}$ Ibid., p. 47.

${ }^{47}$ Edgar S. Brightman, A Philosophy of Religion, Prentice Hall, Nueva York, 1940, p. 102. Brightman también aboga más por el monoteísmo que por la apertura de la antropología filosófica y la ética personalista de Scheler. 
to para Scheler como para Brightman, la religión es un modo de realización cultural y las concepciones culturales, lo que encarnan, tienen una relación directa con el rango de valores que se pueden experimentar.

Scheler sostiene que la persona es la forma más elevada de este devenir del espíritu en la medida en que la persona sublima los impulsos de la vida al espíritu. En el siguiente pasaje, Scheler también menciona al "ser más elevado" como el "fundamento del mundo".

... la sublimación más alta que conocemos, y el devenir humano es la unificación más íntima de las regiones esenciales del mundo. Porque el ser humano unifica todas las regiones esenciales en él, y especialmente la de la vida. Esto vale al menos para sus manifestaciones accidentales, y mucho menos para sus cantidades de distribución. La visión del mundo esbozada aquí hace que desaparezca el conflicto entre una explicación "teleológica" y "mecánica" de la realidad, que prevaleció durante muchos siglos... Este tren de pensamiento no puede detenerse antes incluso del Ser Superior, el fundamento del mundo. También para el Ser, lo que "es a través de sí mismo" y de lo que todo lo demás depende, y en la medida en que el espíritu debe ser asignado a él como uno de sus atributos, tampoco puede tener poder o fuerza como ser espiritual. Más bien, es el otro atributo, la natura naturans en el Ser más elevado, es decir, el "impulso" todopoderoso cargado de imágenes infinitas, que es responsable de la realidad, mientras que el carácter contingente de las entidades nunca se determina unívocamente a través de leyes e ideas esenciales. ${ }^{48}$

En este pasaje, nuevamente, vemos que las fuerzas del espíritu y el impulso co-penetran y se manifiestan con un dinamismo interpenetrante y despliegue regular. La parte enérgica es la intuición fenomenológica que comprende esas esencias de valor, para realizar formas superiores de vida, acción y modos de cultura por encima y en contra de nuestra mera animalidad. Estas fuerzas se unen en el centro de fuerza de la persona. Se interpenetran y se ayudan mutuamente en la operación y refuerzo mutuos ya que la persona es la forma más alta de organización para estos elementos. Sin embargo, notablemente, Scheler intenta una visión intermedia entre un racionalismo cartesiano o escolástico de la metafísica de la sustancia teleológica y un materialismo reduccionista puro. Permítaseme explicar.

La persona no es una sustancia enteramente racional, ni un sistema físico de fuerzas evolutivas. En cambio, la esfera personal se mezcla entre su percepción emotiva y sus esencias de valor y la materialidad encarnada situada y comportándose en el tiempo. En esencia, el compromiso entre enfo-

${ }^{48}$ Scheler, The Human Place in the Cosmos, p. 50. 
ques racionalistas y materialistas se basa en las concepciones anteriores de una racionalidad sentida similar a la visión de participación ontológica grecorromana mencionada anteriormente. La diferencia entre Scheler y la visión grecorromana es que la participación ontológica está dirigida por el amor, no por la racionalidad estoica o platónica estéril que subordina las emociones a la razón.

Además, Scheler está tratando de acomodar la visualización de la persona en un mundo evolutivo del devenir en la tercera concepción anterior. El manejo o dirección del desarrollo de una persona proviene del ejercicio de la libertad de sublimar valores más altos para valores más bajos para que el espíritu motive y anule la suspensión de la vida útil para un propósito más elevado. El propósito es, entonces, inspirador de la ideación y la intuición de las esencias. Por lo tanto, hay una región de esencias, alguna región describió que la esfera personal navega dentro y se da cuenta de todo al mismo tiempo.

Para Scheler, esta fuerza inspiradora y atractiva de la entrega de valores en el Espíritu solo anula las energías reunidas de la vida. Sin las energías reunidas de la vida en nuestros impulsos, las personas no podrían hacer nada, y Scheler hace un argumento especulativo por analogía. Así como el espíritu es impotente en nuestras esferas personales, la misma analogía también está hecha para la esfera personal de Dios. Según Scheler, como persona, Dios como un Ser a través de sí mismo se relaciona con el mundo, y en esa relación nuevamente "hay una tensión primordial entre el espíritu y el impulso" ${ }^{49} \mathrm{Al}$ igual que el experimentador más grande posible de James, Dios tiene la misma estructura que una persona, y el correlato del mundo es aquello con lo que debe relacionarse a través de sí mismo. De esta manera, Dios se vuelve impotente, menos causalmente eficaz que el teísmo clásico en el que Dios se establece en una posición platónica sobre lo que se crea de la nada. Además, el teísmo clásico establece una dependencia asimétrica entre el Creador y lo creado. Para Scheler, esta dependencia limita lo que puede ser la naturaleza, mientras que, en la concepción del proceso de Scheler, el devenir del universo en términos de espíritu e impulso se deja abierto. Existe la posibilidad de crecimiento, no dirigido por ningún conjunto de ideales o por Dios, sino por el hecho de que, si bien Dios puede revelar un ideal a las personas, las personas deben elegir realizar ese valor a expensas de las posibilidades más bajas. De esta manera, tanto el espíritu como el impulso son posibilidades iguales de realizarse específicamente en la convergencia de estas fuerzas en la persona

${ }^{49} \mathrm{Ibid}$. 
humana o en la persona de Dios. La única dependencia se basa en la materialidad encarnada que hace posible la realización del espíritu. En el nivel del espíritu, el acto de sentimiento de amor nos permite sentir la absoluta singularidad y dignidad de una persona. Los valores más altos de lo que Scheler llama valores sagrados son la realización de valor más alta posible.

A medida que entiendo estos elementos de entrega en las personas, y en el esquema más amplio del fundamento del Ser, el espíritu y la impulsión se convierten en la presencia dominante de todo lo que existe. De esta manera, el panenteísmo es que Dios es una persona en todo lo que es y está por ser, sin embargo, estos elementos del espíritu y el impulso no son contrarios tanto como son formas para todo lo que es como una persona es y aún debe ser. En otras palabras, la realidad deviene en el espíritu y el impulso de Dios tan fácilmente, ya que también es una convivencia compartida en el centro de actos de las personas humanas. De hecho, devenir es esta colaboración en nosotros, a través de nosotros, y en nosotros en espíritu y a través del espíritu que constituye y constituye la realidad. La realidad se convierte en estas fuerzas subyacentes a la experiencia y simultáneamente como experiencia. El espíritu y el impulso se compenetran e interpenetran, desarrollándose activamente. De alguna manera, este devenir es pasivo y de otras formas directamente experimentado como activo. Por esta razón, me parece más apropiado rechazar este devenir como un tipo de dualismo, pero pensar en el espíritu y el impulso de la misma manera que los teístas del proceso han insistido en un tipo de teísmo dipolar.

\section{Pensando en el devenir como teísmo dipolar}

En el teísmo dipolar, el devenir potencial de Dios existe en un estado de posibilidad, aún no como los valores invocados en el espíritu de Scheler. En la otra mitad, las energías de la vida en la materialidad del impulso y las realidades ya se han convertido y actualizado en un conjunto de posibilidades. Dado que el Dios de Scheler deviene en la persona y en todos nosotros al mismo tiempo, todo lo que es como persona es y aún está por ser es una expresión que combina el hecho de que los aspectos del Dios panenteísta ya están en el tiempo, ya están surgiendo y retrocediendo en el tiempo y la experiencia. Por esta razón, establecer paralelismos con el último capítulo de Alfred North Whitehead en Proceso y realidad debería constituir trabajo futuro y compro- 
miso con los escritos de Scheler. ${ }^{50}$ No intentaré un análisis tan exhaustivo, aunque muy necesario. Por ahora, simplemente esbozaré una forma en la que puede verse este compromiso, aunque deberían seguirse tratamientos más exhaustivos al resaltar esta característica intrigante y dirección interpretativa que el pensamiento de Scheler podría tomar en futuros estudios. ${ }^{51}$

En Whitehead, cada entidad real, la unidad más atómica del universo, ya sea que estemos hablando de pequeños soplos de existencia o de personas, todos tienen un polo físico y un polo mental. Estos aspectos no son las entidades en sí mismas, sino meramente aspectos de ellas de la misma manera que yo llamo que las formas de entrega personal del espíritu y el impulso pueden tender a pesar de que ambos elementos están constantemente co-penetrando. Como cualquier otra entidad, Dios es una entidad real con un polo físico y un polo mental, que él llama la naturaleza consecuente y la naturaleza primordial. Estos dos aspectos de Dios existen como partes inseparables de Dios, pero se desarrollan en relación entre sí. La naturaleza primordial es como el espíritu de Scheler. Expresa lo que podría ser el conjunto de posibilidades en que la realidad puede crecer. En contraste, la naturaleza consecuente expresa el hecho sólido de lo que ha sido, la materialidad y la determinación que da forma a la posibilidad. El impulso de Scheler, sin embargo, es un poco diferente, al parecer, de Whitehead. Para empezar, la naturaleza consecuente de Whitehead se refiere a la inmortalidad objetiva de toda determinación que se recuerda y su interacción es lo que limita la expresión pura de lo que es posible en la naturaleza, y el impulso en las personas se refiere a la excitación y liberación de impulsos psíquicos de las personas. Sin embargo, de manera limitada, el impulso puede indicar los procesos vitales en Whitehead de los que depende la posibilidad del espíritu.

\section{Conclusión}

En este ensayo, he atravesado aspectos de la tardía metafísica y antropología filosófica de Scheler. El objetivo ha sido comprender cómo cambió el pensamiento de Scheler, qué dirección tomó y cómo comprender mejor los ele-

${ }^{50}$ Alfred North Whitehead, Process and Reality, Corrected Edition, Free Press, Nueva York, 1978, pp. 342-351.

${ }^{51}$ El primero en la literatura que destaca la necesidad de que los Schelerianos presten atención a Whitehead es Randall Auxier, quien en el siguiente artículo no revela tanto a Scheler como un teísta dipolar, sino que exige la explicación de Scheler del sentimiento intencional para corregir aspectos del pensamiento de Whitehead. De manera irónica, pido a la comunidad de Scheler que preste atención a Whitehead como una forma de corregir el dualismo de Scheler. 
mentos basados en procesos de su metafísica posterior. Como Scheler es recordado principalmente por la habilidad de Juan Pablo II, Scheler tiende a ser preservado y leído a través de su compromiso tentativo con su catolicismo. La fenomenología es un camino hacia los valores de lo Santo y refuerza, por así decirlo, los dogmas de la teología religiosa católica. Esta tendencia es desafortunada y totalmente errónea cuando el alcance de la vida de Scheler se ve en su totalidad.

Además, no he leído los argumentos de Peter Spader en su totalidad, ni les he dado el trato que se merecen. Dado que su Scheler's Ethical Personalism: Its Logic, Development, and Promise (2002) fue el penúltimo compromiso académico en inglés sobre la ética de Scheler, aparte de Material Value Ethics: Max Scheler and Nicolai Hartmann (2011), de Eugene Kelly, los argumentos de Spader, aunque los veo como incorrectos, deben ser atendidos con mayor atención. En este artículo, no tuve tiempo de revisar sus interpretaciones tanto de las razones de esta interpretación dualista como de los problemas de la lectura del dualismo entre Geist y Drang que cortan las líneas del interaccionismo que encontramos en Descartes. En cambio, solo he indicado en espíritu con Whitehead que esa filosofía podría leerse como un tipo de monismo dipolar en el que tanto el espíritu como el impulso son aspectos revelados de cómo las personas (tanto humanas como de Dios) se revelan a sí mismas y cómo y por qué esta realización de la entrega de uno mismo también constituye el sentido de la realidad. De esta manera, mi lectura ha comprado las advertencias de Whitehead contra los relatos bifurcados de la realidad, esencialmente el anti-dualismo de Whitehead, aunque no he presentado ningún argumento explícito para tal interpretación. Sólo he ofrecido una especulación.

Sin embargo, lo que revela la metafísica posterior de Scheler es la necesidad de pensar más allá de las categorías de ortodoxia religiosa y teológica. Varios dogmas nos roban el imaginario cultural y político para concebir lo Divino más allá de la tradición. En mi país, Estados Unidos, el cristianismo se moviliza bajo la bandera de ideologías capitalistas que socavan directamente la santidad de las personas. De esta manera, el cristianismo está continuamente armado, y aunque tengo muchos - y a veces yo mismo- - adheridos a los principios cristianos del amor agápico en el ejemplo de Cristo y atraídos por la política del reverendo Dr. Martin Luther King, Jr., me temo que el cristianismo liberal y progresista nunca es suficiente, nunca es capaz de movilizar las acciones y reformas justas necesarias para restringir la pandemia global del cambio climático que engendra Estados Unidos o la violencia estructural de 
la desigualdad de ingresos a nivel nacional. Consumimos la energía del mundo varias veces, y nos explotamos mutuamente y a la naturaleza en nombre de Dios constantemente. Bajo el manto de Dios, Estados Unidos lanza ataques en todo el mundo en lo que para muchos es una guerra religiosa a pesar de los muchos civiles inocentes que mueren en nuestra cruzada obsesionada por Dios contra el sustantivo abstracto de "terrorismo". Así, los cristianos en los Estados Unidos se olvidan en gran medida de vaciarse del amor kenótico para recibirlo de otro. Por lo tanto, hace tiempo que pensaba que un desafío cultural masivo a la ortodoxia cristiana debería venir de lo que me atrevo a llamar teísmo especulativo. ${ }^{52}$

Así como los materialistas especulativos en la filosofía continental quieren restablecer un retorno a las ontologías amigables con los objetos, también quiero socavar la ortodoxia de nuestras nociones abrahamicas de Dios. Llamo a los filósofos a imaginar las diferentes formas en que Dios puede ser concebido. Llamo a los filósofos a explorar esos sistemas conceptuales, recuperando los idealismos, panenteismos y panteísmos del pasado para extraerlos de lo que podría servir y beneficiar pragmáticamente nuestra experiencia compartida, así como analizar sus defectos especulando sobre las nuevas formas que puede tomar lo Divino. De esta manera, el trabajo de Scheler es primordial, ya que es uno de los últimos ejemplos de teísmo especulativo (junto con Whitehead) que socava y estira el concepto de Divinidad y Naturaleza para oponerse directamente a los conceptos contaminados de Dios en el cristianismo y reavivar nuevas teologías políticas que incorporar nuestras obligaciones mutuas a nivel nacional, internacional y al planeta que todos llamamos hogar. En ninguna parte es este trabajo más importante que los Estados Unidos que lidera el mundo en un sistema económico insostenible y nos ciega a otros fines inmorales a los que muchas hermanas y hermanos de nuestra familia humana están afectados por actitudes impersonales de interés propio en la economía y el imperialismo militar.

\section{Bibliografía}

Brightman, E.S., 1940, A Philosophy of Religion, Nueva York, Prentice Hall, Nueva York.

${ }^{52}$ La profesora Angela Roothaan de la Universidad libre de Amsterdam me ha recordado que mi llamado al teísmo especulativo no presta atención a los muchos enfoques postmetafísicos de Dios que pueden extraerse de conceptos más allá de lo que yo llamo Abrahamico. Estos enfoques postmetafísicos pueden ejemplificarse en analogía, como la preocupación de la teología de la liberación por el Dios pobre. 
Frings, M. S., 2001, The Mind of Max Scheler, Marquette University Press, Milwaukee.

Kelly, E., 2011, Material Ethics of Value: Max Scheler and Nicolai Hartmann, Springer, Dordrecht.

Scheler, M. F., 2006, Formalism in Ethics and Non-Formal Ethics of Value: A New Attempt Toward the Foundation of an Ethical Personalism, Northwestern University Press, Evanston.

, 2009, Human Place in the Cosmos, Northwestern University, Evanston.

- 2009, On the Eternal in Man, Transaction Publishers, New Brunswick y Londres.

Spader, P., 2002, Scheler's Ethical Personalism: Its Logic, Development, and Promise, Fordham University Press, Nueva York.

Whitehead, A.N., 1978, Process and Reality, Corrected Edition, Free Press, Nueva York. 